\title{
Electrodeposition polyaniline nanofiber on the PEDOT:PSS-coated SiNWs for high performance supercapacitors
}

Xiaojuan Shen (D 1000004296@ujs.edu.cn )

Jiangsu University

Xuan Zhang

Jiangsu University

Tongfei Wang

Jiangsu University

Songjun Li

Jiangsu University

Research Article

Keywords: SiNWs, Micro-supercapacitors, Electrodeposition, Polyaniline, PEDOT:PSS

Posted Date: April 8th, 2021

DOI: https://doi.org/10.21203/rs.3.rs-402012/v1

License: (c) (i) This work is licensed under a Creative Commons Attribution 4.0 International License.

Read Full License 


\section{Abstract}

In this article, a novel silicon-based electrode was designed with the facile solution methods. With the modification of highly conductive PEDOT:PSS layer on the SiNWs by the spin-coated method (SiNWsPSS), three-dimensional (3D) porous network polyaniline nanofibers (PANI) film was uniformly electrodeposited on the silicon surface (SiNWs-PSS@PANI). The sheet resistances of the PEDOT:PSS layer with different surfactants as well as the deposition time of the PANI were investigated. After optimization, the fabricated SiNWs-PSS@PANI electrode displayed high capacitance about $301.71 \mathrm{mF}$ $\mathrm{cm}^{-2}$ at the current density of $1 \mathrm{~mA} \mathrm{~cm}-2$, which enhanced $\sim 29$ fold comparing to $10.18 \mathrm{mF} \mathrm{cm}$ 2 of electrode without the PEDOT:PSS layer between the SiNWs and PANI (SiNWs@PANI), outperforming most values of the reported silicon-based electrodes. The electrode designed in this paper provides a new idea to fabricate high-performance of silicon-based micro-supercapacitors with the simple and low-temperature method.

\section{Full Text}

This preprint is available for download as a PDF.

\section{Figures}
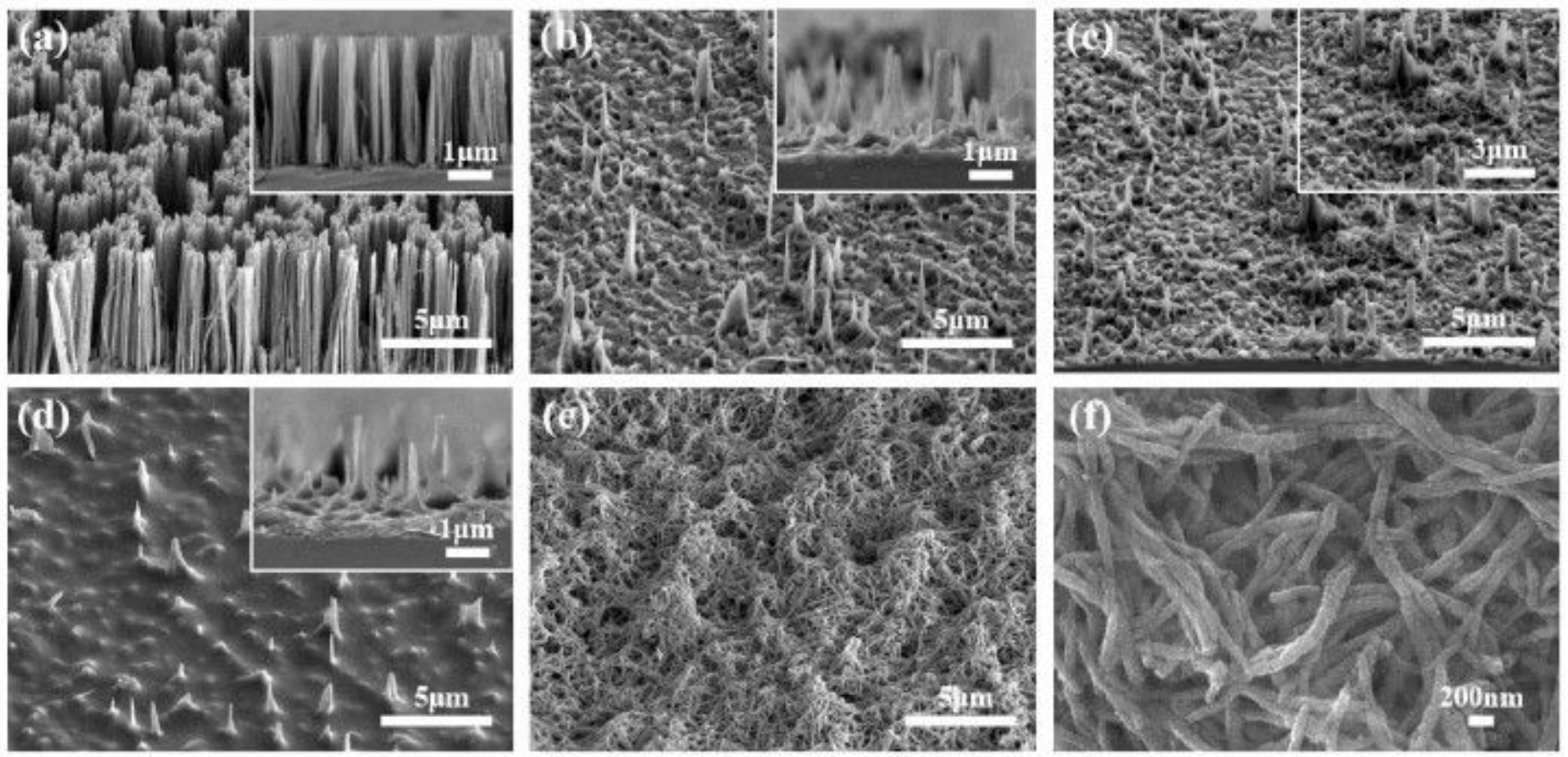

Figure 1

SEM images of different silicon substrates. a SiNWs synthesized with MACE method for $60 \mathrm{~min}$, b SiNWs treated by $\mathrm{PCl} 5$ solution for $2 \mathrm{~h}$. Insets are cross-section corresponding images. c PANI electrodeposited on the pristine SiNWs substrate using the $\mathrm{Ag}$ as the rear electrode for $10 \mathrm{~min}$, the inset shows the image 
at high magnification, d SiNWs-PSS. Inset is cross-section image, e and f different magnification images of SiNWs-PSS@PANI with the deposition time of 5 min.

(a)
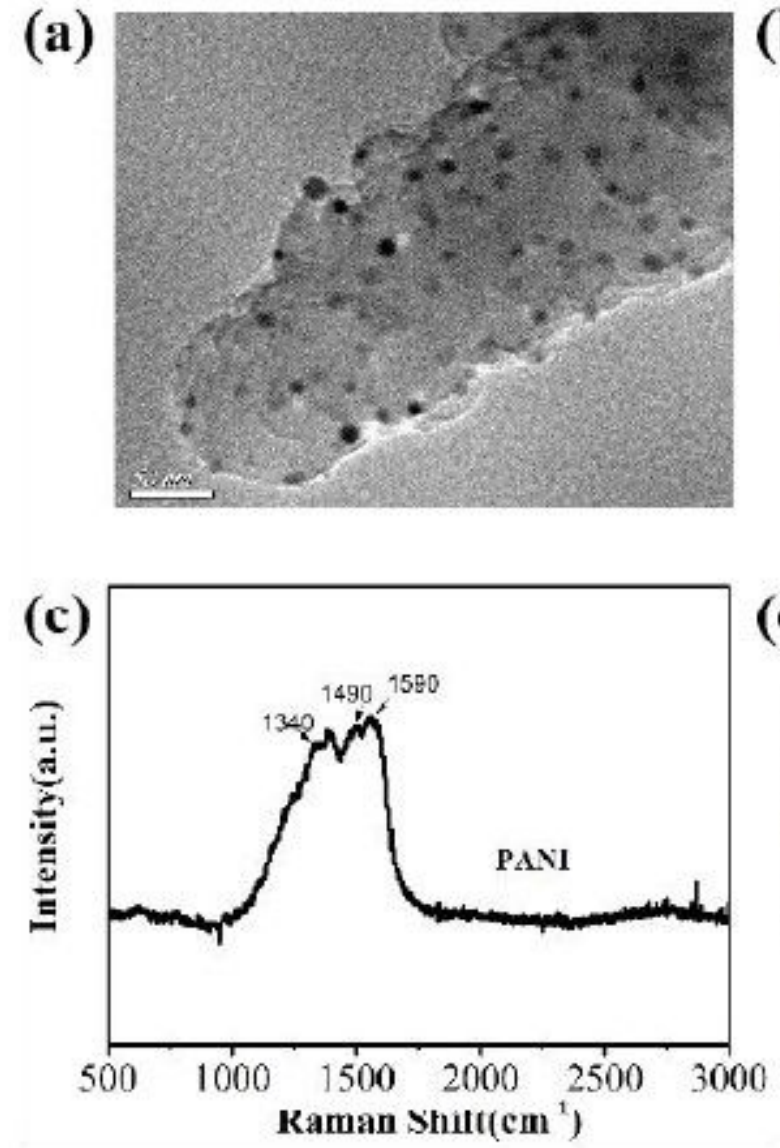
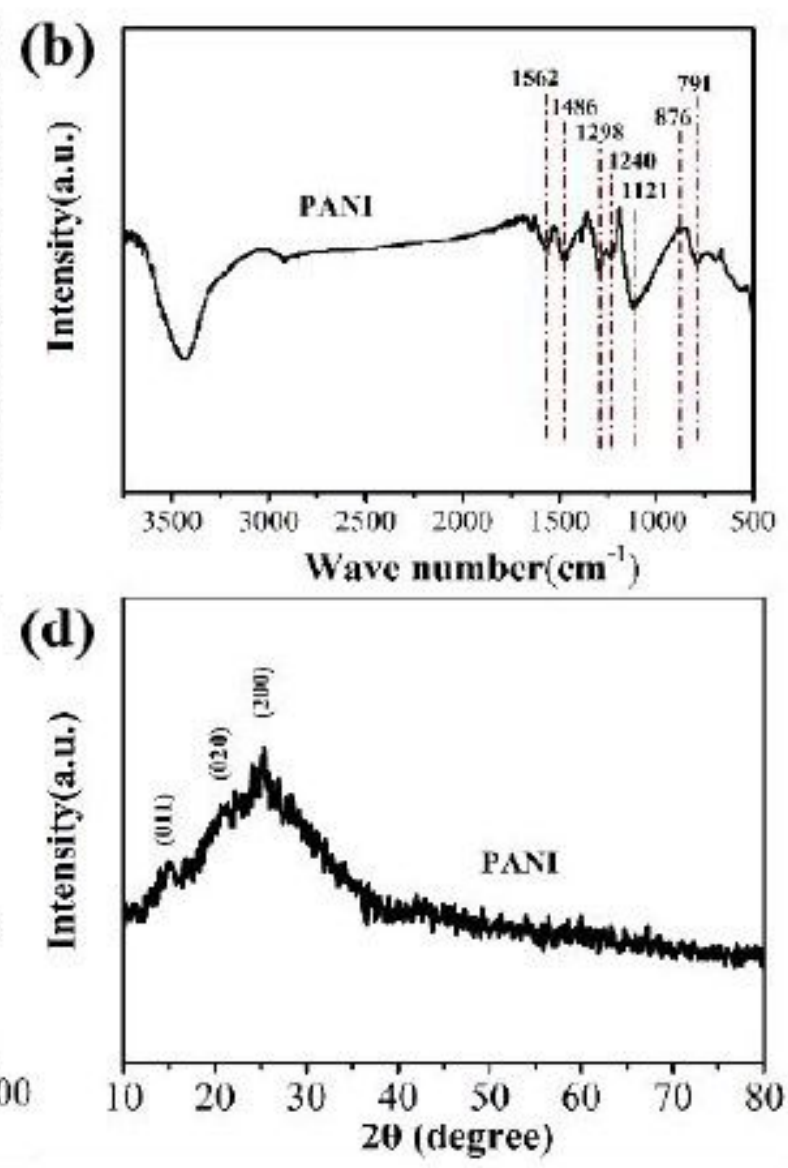

Figure 2

a TEM image of electrodeposited polyaniline nanofibers, b FTIR spectra of PANI, c Raman spectra of PANI and $d X R D$ patterns of PANI. 

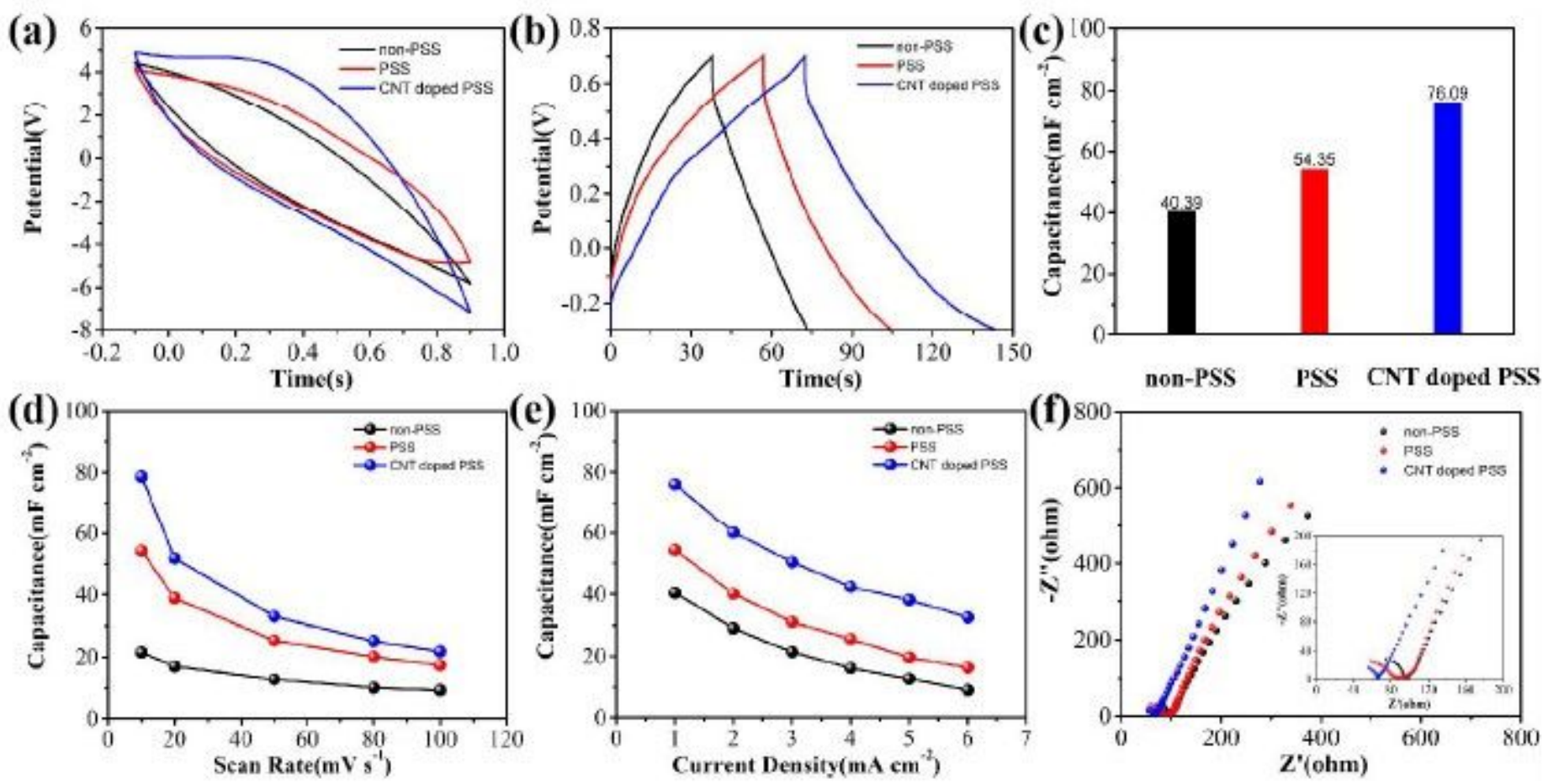

Figure 3

Electrochemical performance of the SiNWs-PSS@PANI electrodes with different top layer. a CV curves at the scan rate of $50 \mathrm{mV} \mathrm{s}-1, \mathrm{~b}$ GCD measurement at the current density of $1 \mathrm{~mA} \mathrm{~cm}-2$, c areal capacitances at the current density of $1 \mathrm{~mA} \mathrm{~cm}-2, \mathrm{~d}$ areal capacitance at different scan rates, e areal capacitance at different current densities and $f$ Nyquist impedance plots at the open circuit voltage. The electrodeposition time is $5 \mathrm{~min}$.
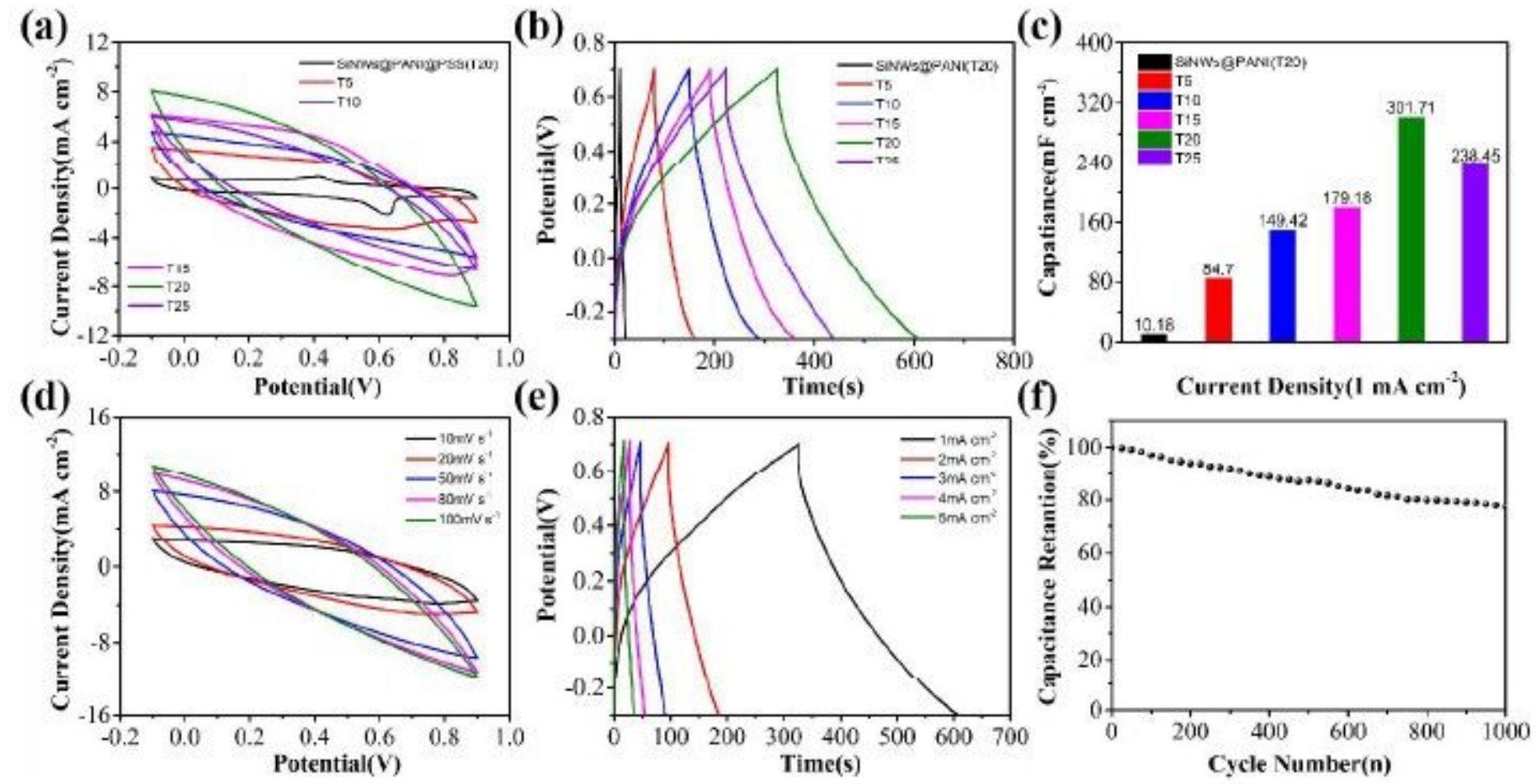
Figure 4

a CV curves at a scan rate of $50 \mathrm{mV} \mathrm{s}-1, \mathrm{~b}$ GCD curves at $1 \mathrm{~mA} \mathrm{~cm}-2, \mathrm{c}$ areal capacitance of different electrodeposition time extracted from the GCD curves, d CV curves at various scan rates, e GCD curves at different current densities and $\mathrm{f}$ Cyclic stability at $100 \mathrm{mV} \mathrm{s}-1$ of the electrode.
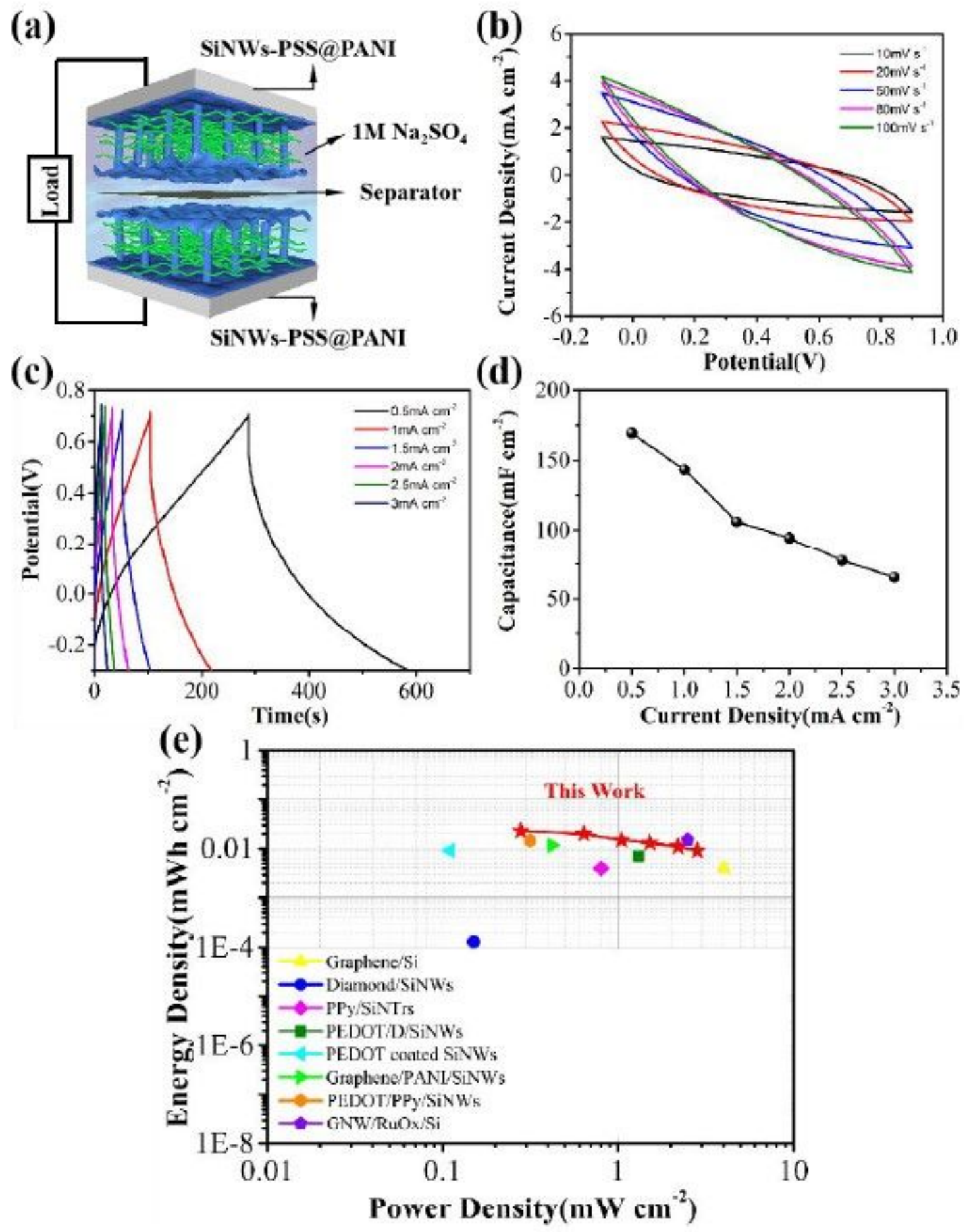

Figure 5

a Schematic diagram of a symmetric supercapacitor based on SiNWs-PSS@PANI electrodes, b CV curves at various scan rates, c GCD curves at different current densities, $d$ areal capacitance at different current 
densities and e Ragone plots of MSCs with other reported values for comparison.

\section{Supplementary Files}

This is a list of supplementary files associated with this preprint. Click to download.

- GraphicalAbstract.jpg

- Supplementarydata.pdf 